\title{
Management Algorithm for Cystic Pancreatic Lesions
}

\author{
Georg Beyer $^{a} \quad$ Elisabetta Goni $^{a} \quad$ Marlies Köpke ${ }^{a} \quad$ Jan G. D'Haese ${ }^{b} \quad$ Jens Werner ${ }^{b}$ \\ Jörg Schirra ${ }^{a}$ Julia Mayerle $^{a}$
}

${ }^{a}$ Medical Department II, University Hospital, Ludwig Maximilians-University, Munich, Germany;

${ }^{b}$ Department of Surgery, University Hospital, Ludwig Maximilians-University, Munich, Germany

\section{Keywords}

Pancreatic cyst surveillance .

Intraductal papillary mucinous neoplasm, IPMN .

Fukuoka guidelines - Worrisome features .

High-risk stigmata

\section{Summary}

Cystic pancreatic lesions are common findings in an aging society due to an increasing availability of highresolution cross-sectional imaging. Although the overall prevalence of malignancy and the rate of malignant conversion are low, especially mucinous pancreatic cystic lesions such as intraductal papillary mucinous neoplasm and mucinous cystic neoplasm harbor significant malignant potential depending on their morphology and size. Recently updated guidelines recommend sophisticated algorithms for initial workup and surveillance based on individual characteristics of the cystic lesion and the patient, thus weighing the lifetime risk for malignancy against the adverse event rate of potentially curative surgery in the light of number and location of cystic lesions, age of the patient, comorbidities, and the resulting life expectancy as well as the effect of repeated follow-up examinations on the patient's quality of life. This article summarizes recommendations from available guidelines and proposes a pragmatic approach to the clinical management of pancreatic cystic lesions.

(c) 2018 S. Karger GmbH, Freiburg

\section{Introduction: Whom to Treat, Whom to Observe, and Whom to Forget}

Cystic pancreatic lesions are an increasingly common entity, thus posing a clinical and socioeconomic challenge [1]. From pop- ulation-based studies we have learned that especially small cystic lesions are a very common entity in the elderly and that the increasing accuracy and availability of magnetic resonance imaging (MRI) potentiates the number of incidental diagnoses [2]. They are therefore both a disease of age and of advancing technology. We have also learned that the vast majority of these incidental cystic lesions is small and that, from an epidemiological point of view, the risk of developing pancreatic malignancy is small [2].

The question whether to treat, to observe, or to ignore a cystic pancreatic lesion depends on two simple questions; however, finding the answer to these can be difficult: i) Is the lesion causing symptoms? ii) What is the correct clinical entity?

Symptomatic cystic lesions require treatment [3]. Whether a symptom, e.g. abdominal pain, can be attributed to the cystic lesion is not always easy to determine. We have learned that most cystic lesions are asymptomatic even if abdominal symptoms might have triggered initial imaging, which led to its diagnosis. The mode of treatment consecutively depends on the type of cystic lesions, the kind of symptoms, and the patient's condition. If the lesion has malignant potential (intraductal papillary mucinous neoplasm (IPMN), mucinous cystic neoplasm (MCN), solid pseudopapillary neoplasia (SPN), cystic neuroendocrine tumor) and the patient is fit, treatment will most likely be surgical. If there is no malignant potential (serous cystic adenoma (SCN), pseudocysts) or the patient is unfit for surgery, additional medical or endoscopic treatment needs to be discussed. It has to be kept in mind that these decisions should not be made without the patient and that a multidisciplinary team approach should be followed.

In all asymptomatic cystic lesions, establishing a diagnosis is critical for any subsequent management decision, as long as the patient is fit for surgery. In patients who are not suited for surgery and do not have symptoms, incidental cystic pancreatic lesions do not require follow-up as it will not influence the clinical course [3, 4]. In all other cases, the major question is whether or not the lesion harbors risk factors for malignancy or whether it has a potential for malignant development in the future. In all cystic lesions

\section{KARGER}

(๑) 2018 S. Karger GmbH, Freiburg

Fax +497614520714
Prof. Dr. med. Julia Mayerle 
with high-risk stigmata for malignancy (table 1), surgery needs to be considered by a multidisciplinary team. For all other cystic lesions with malignant potential (IPMN, MCN, SPN), further evaluation and surveillance are usually recommended. In young patients or if a singular lesion is suitable for distal pancreatectomy, upfront surgery should be discussed with the patients even if there are no worrisome features or high-risk stigmata present in order to avoid a lifelong follow-up [4]. If the patients' history as well as combined diagnostic yield of imaging and cyst fluid analysis supports the diagnosis of pancreatic pseudocyst or SCN, surveillance is not recommended due to a lack of malignant potential and due to the benign course of the disease [5].

Whether or not cystic lesions measuring less than $5 \mathrm{~mm}$ in diameter should be followed is unclear. Modern MRI and endoscopic ultrasound (EUS) techniques frequently detect very small lesions; however, it is almost impossible to make a conclusive diagnosis [2] Since the risk of malignant transformation in those patients is extremely low [2], a pragmatic approach would include a one-time MRI control after 6 months and discharge from follow-up if unchanged in appearance and without symptoms.

\section{Establishing a Diagnosis: Role of Cross-Sectional Imaging and Endoscopic Ultrasound}

The diagnostic accuracy of multi-detector computed tomography (MDCT) and MRI with magnetic resonance cholangiopancreatography (MRCP) for pancreatic cystic lesions is reportedly similar. However, we recommend the use of MRI with MRCP for the diagnosis and surveillance of pancreatic cysts in terms of crosssectional imaging as it has a higher detection rate (19.9 vs. $2.6 \%$ ) with a similar specificity and sensitivity for malignant transformation of 0.81 (95\% confidence interval (CI) $0.71-0.88$ ) versus 0.76 (95\% CI 0.81-0.99) [6, 7]. Moreover, MRI can detect smaller additional lesions which might have been missed on CT but will influence the decision making process for further management. Spatial resolution of MRI is also higher compared to MDCT, and dedicated ductal imaging via MRCP might help identifying worrisome features (table 1) in lesions undergoing surveillance. In addition, MRI does not use ionizing radiation, thus making it more suitable for long-term surveillance. Whether or not there is a role for positron emission tomography (PET)-CT, PET-MRI, or transabdominal ultrasound for the diagnosis and surveillance of pancreatic cystic lesions is unclear. MDCT has a potential diagnostic advantage in case of calcifications of the cystic lesions and/or when the pancreatic parenchyma is present and is often required for accurate staging when malignancy is suspected or proven and when vascular involvement needs to be excluded.

EUS \pm fine needle aspiration (FNA) is recommended in adjunction to cross-sectional imaging in cases of diagnostic uncertainty regarding the type of cystic lesion and for risk stratification $[3,4]$. Data on its diagnostic accuracy are conflicting as it is more operator-dependent [8]. Therefore, it should not be used as the only modality but to complement MRI. EUS is minimally invasive and the
Table 1. Overview of worrisome features and high-risk stigmata (according to [4])

\begin{tabular}{ll}
$\begin{array}{l}\text { Worrisome features } \\
\text { Diameter of } \geq 3 \mathrm{~cm} \\
\text { Enhancing mural nodule }>5 \mathrm{~mm} \\
\text { Thickened/enhancing walls } \\
\text { Main duct } 5-9 \mathrm{~mm} \\
\text { Abrupt caliber change of main pancreatic duct } \\
\begin{array}{l}\text { Lymphadenopathy } \\
\text { Serum level CA } 19-9 \text { elevated } \\
\text { Growth }>3 \mathrm{~mm} / 12 \text { months; } 5 \mathrm{~mm} / 24 \text { months }\end{array}\end{array} \quad \begin{array}{l}\text { indication for EUS } \pm \\
\text { FNA and closer sur- } \\
\text { veillance; if confirmed } \\
\text { definite mural nodule(s) } \\
>5 \mathrm{~mm} \text {, main duct } \\
\text { features suspicious for } \\
\text { involvement, or cytolo- } \\
\text { gy suspicious or positive } \\
\text { for malignancy, consider } \\
\text { surgery }\end{array}$ \\
\hline
\end{tabular}

High-risk stigmata

Obstructive jaundice caused by cystic lesions

Enhancing mural nodule $\geq 5 \mathrm{~mm}$

consider surgery

Main duct $\geq 10 \mathrm{~mm}$

EUS = Endoscopic ultrasound; FNA = fine needle aspiration.

adverse event rate is low. According to the latest revision of the international consensus guideline, it plays an additional role as a step-up diagnostic test if cross-sectional imaging picks up features worrisome for malignancy in branch-duct IPMN (BD-IPMN), i.e. in detecting definitive mural nodules $>5 \mathrm{~mm}$, definitive main duct involvement, and suspicious cytology from FNA [4].

Cyst fluid analysis from EUS-FNA can help to distinguish mucinous lesions (MCN, IPMN) from other types. The only established and routinely recommended biomarker is carcinoembryonic antigen (CEA). It has a sensitivity and specificity of 63\% (95\% CI 59-67) and 93\% (95\% CI 90-95), respectively, for identifying IPMNs and MCNs in a meta-analysis of 18 papers $[9,10]$. The recommended cutoff for cyst CEA is $>192-200 \mathrm{ng} / \mathrm{ml}$ which results in about $80 \%$ accuracy for the diagnosis of mucinous lesions [3, 4 , 10]. Amylase or lipase levels are elevated in lesions communicating with the pancreatic ductal system (BD-IPMN, pseudocysts) and can be measured in addition to CEA; however, they are less specific. SCNs are low in both CEA and amylase/lipase levels.

Cystic lesions with strong evidence for main duct involvement on B-mode EUS (main duct IPMN (MD-IPMN) or mixed-type IPMN) with pancreatic duct dilatation $>10 \mathrm{~mm}$ should not undergo EUS-FNA for cyst fluid analysis as it most likely does not influence clinical decision making and has a high risk of causing pancreatitis episodes [11].

\section{Risk Stratification}

The risk for concomitant malignancy or malignant transformation of mucinous cysts and SPN in the future depends on the type of cyst and can be additionally evaluated by clinical and morphological criteria, classified as high-risk stigmata and worrisome features by the International consensus guideline (aka Fukuoka guideline, formerly Sendai guideline) $[4,12,13]$, although formally established only for BD-IPMN (table 1). The clinical challenge lies in 
weighing the lifetime risk for malignancy against the adverse event rate of potentially curative surgery in the light of number and location of cystic lesions, age of the patient, comorbidities, and the resulting life expectancy as well as the effect of repeated follow-up examinations on the patient's quality of life.

IPMNs are potentially premalignant neoplasms. They can be subdivided into MD-, BD-, and mixed-type IPMNs. MD-IPMN is defined by segmental or diffuse dilatation of the main pancreatic duct over $5 \mathrm{~mm}$. In contrast, BD-IPMNs lack main duct dilatation but communication with the main duct is common. Mixed-type IPMNs, as expected, show both involvement of the main pancreatic duct as well as side branches but are clinically treated like MDIPMNs. MCN are of non-ductal origin and occur mostly in young to middle-aged women while their malignant potential is similar to BD-IPMN.

\section{$B D-I P M N / M C N$}

In BD-IPMN and MCN, criteria that indicate the presence of high-grade dysplasia or invasive carcinoma and that should trigger surgical evaluation are presence of mural nodules $>5 \mathrm{~mm}$ that can be reproduced on EUS, a serum carbohydrate antigen (CA) 19-9 > $37 \mathrm{U} / \mathrm{l}$, and cytology results positive or highly suspicious of malignancy. Obstructive jaundice caused by a cystic lesion located in the head of pancreas is regarded as a clinical high-risk stigma. Whether new-onset diabetes or worsening glycemic control can play a role in detecting high-risk mucinous lesions is currently debated. Cyst fluid analysis for CEA has no value in distinguishing the presence of high-grade dysplasia or cancer. So-called worrisome features include pancreatitis, enhancing mural nodules or thickened cyst walls on MRI, a main pancreatic duct of 5-9 $\mathrm{mm}$, abrupt caliber changes of the main pancreatic duct, a cyst size $>3 \mathrm{~cm}$, and cyst growth of $>5 \mathrm{~mm} / 2$ years or $3 \mathrm{~mm}$ in 1 year. If any of those are present, EUS \pm FNA should be performed to exclude high-risk stigmata (not in MD-IPMN), and surveillance needs at least to be intensified.

Even in the absence of other concerning features, most centers will consider surgery if a BD-IPMN or MCN reaches a size of $3 \mathrm{~cm}$ or more. This is supported by a systematic review including 644 cysts from 6 studies (odds ratio (OR) for high-grade dysplasia or pancreatic cancer of 2.97 (95\% CI 1.82-4.85)) [14] as well as by a systematic review and meta-analysis of 1,058 IPMNs from 16 studies (OR 62.4; 95\% CI 30.8-126.3) [15]. A recent analysis of 211 resected MCNs showed that in only 5 cases malignancy occurred in lesions smaller than $4 \mathrm{~cm}$ and that all lesions with high-grade dysplasia or cancer were associated with worrisome features on preoperative imaging [16]. This could in turn lead to the conclusion that MCNs smaller than $3 \mathrm{~cm}$ and without any other features of concern are safe to observe. Notably, in the rare case of MCN in a male patient, malignant transformation seems to occur more often. If cystic lesions change rapidly in size, some evidence points towards a higher risk of malignancy [3].

\section{MD-/Mixed-type IPMN}

In MD-/mixed-type IPMN, dilatation of the main pancreatic duct $>10 \mathrm{~mm}$ is a high-risk stigma for malignancy and should lead to strong consideration of a surgical therapy. In patients with a main pancreatic duct measuring between 5 and $9 \mathrm{~mm}$, the finding should be treated as a worrisome feature and the patient can undergo further evaluation by EUS and close surveillance. Notably, other causes for main pancreatic duct dilatation need to be ruled out.

In general, the risk of high-grade dysplasia or malignancy in IPMN varies greatly across the literature and seems to be uniformly higher in a series of surgical cases (BD-IPMN 6.1-47.9\%, MDIPMN 36-100\%) compared to the annual progression rate in prospective or retrospective cohorts that underwent surveillance (BDIPMN 1.4-6.9\%) [3, 4]. In the first place, this seems to be a strong indicator of a selection bias towards symptomatic or otherwise clinically and radiologically remarkable patients enriched in surgical cohorts. This could also mean that despite objective indicators for close surveillance and surgical evaluation outlined in recent guidelines, the clinical judgement of the treating physicians plays a critical role in choosing the right strategy.

\section{SPN}

SPNs are rare and occur in young women. The risk of malignancy is moderate $(4.6 \%)$, but the requirement of otherwise lifelong surveillance makes surgery the preferred mode of treatment. As always, it should be carried out at a high-volume center to avoid adverse outcome $[17,18]$.

\section{Management Algorithm}

All patients with newly diagnosed cystic pancreatic lesions should be referred to a high-volume pancreatic center for initial workup, establishment of a diagnosis, and plan for management [3, 4]. Centers should be able to provide expert opinion from gastroenterology, surgery, and radiology, and EUS with FNA as well as a current standard MRI facility should be available.

So far, no study could show a survival benefit for patients undergoing cyst surveillance. However, evidence suggests that cystic lesions do exist for a period of years before malignant transformation occurs. Therefore, detection of high-risk stigmata for malignancy can potentially lead to resection in early stages of malignant development, thus improving the chance of curative surgery. The overall goal is to identify cystic lesions at a stage before invasive cancer occurs and to admit these patients to surgery while preventing all unnecessary procedures.

After a cystic lesion has been detected and adequate imaging has been obtained, a decision between immediate treatment, followup, or discharge is made based on the working diagnosis, symptoms, and the condition of the patient (fig. 1). If a patient enters a surveillance program, usually the frequency of follow-up imaging depends on the size of the lesion and the presence of worrisome features. However, the international consensus guideline recommends a first re-evaluation after 3-6 months for every lesion to ensure that dramatic changes will not be missed. Although this probably leads to overtreatment of smaller cystic lesions, it seems to be a pragmatic approach for most patients. The recently published 
Fig. 1. Model for initial management after cyst discovery and how to make a decision on whom to treat immediately, whom to observe, and whom to discharge from surveillance.

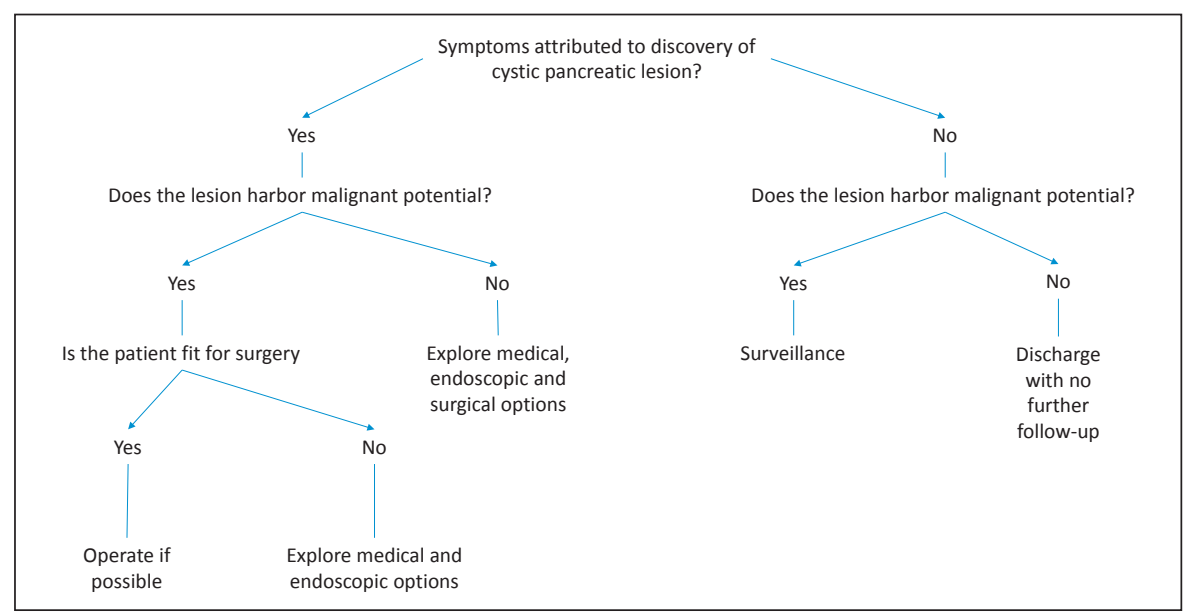

Fig. 2. Proposed management algorithm for surveillance of asymptomatic mucinous cystic lesions of the pancreas incorporating recommendations from the American College of Gastroenterology clinical guideline [3] and revisions of the international consensus

Fukuoka guidelines [4].

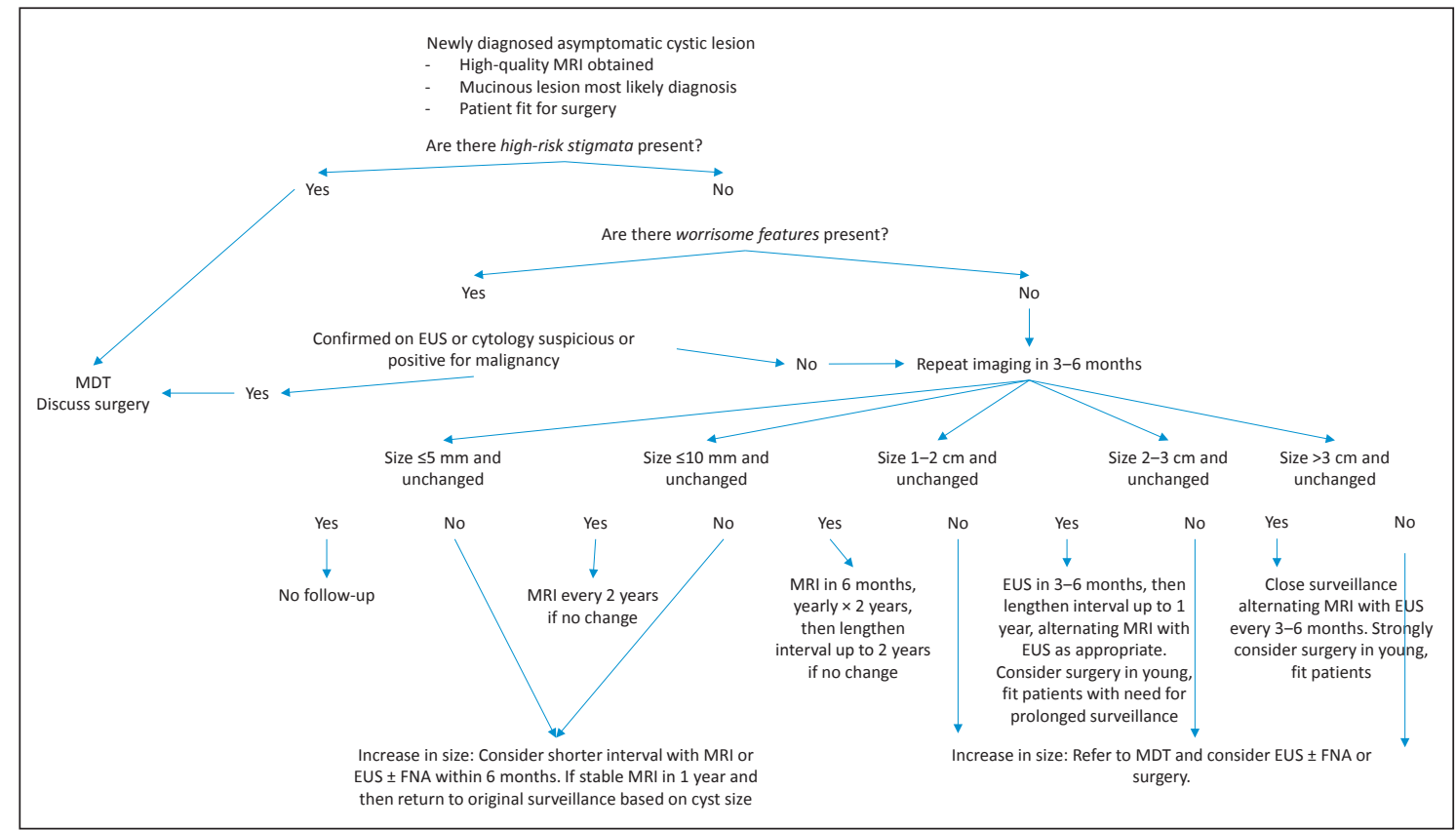

American College of Gastroenterology guideline recommends imaging at 6 months only for cystic lesions $>2 \mathrm{~cm}$ or if concerning features were present at initial presentation. In most cases MRI will be used for follow-up; however, if EUS was initially conducted and the lesion has been well characterized, repeat EUS seems to be adequate as well, depending on local preference.

If a lesion presents as stable in size and appearance during follow-up, the interval for imaging can be lengthened as indicated in figure 2. Likewise, if worrisome features or high-risk stigmata occur during follow-up, intervals need to be shortened, EUS-FNA considered, and even surgery re-evaluated. Any relevant change should trigger referral to experienced pancreatic centers and discussion among a multidisciplinary team of experts in the field. If new lesions occur during the follow-up period, they should be treated as new independent cystic lesions. In addition to cyst evaluation, pa- tients need to be regularly evaluated for comorbidities that might make them unsuitable for surgery as this would change the surveillance strategy (as mentioned above).

Currently, there is no evidence that supports to stop surveillance at a certain age or after several years with stable appearance of cystic lesions. The decision has to be made individually as well as based on the patients' condition and their personal preference. Although cystic pancreatic lesions are a clinical challenge with pandemic dimensions, management can only be conducted on an individual level.

\section{Disclosure Statement}

None declared. 


\section{References}

1 Budde C, Beyer G, Kühn J-P, Lerch MM, Mayerle J: The clinical and socio-economic relevance of increased IPMN detection rates and management choices. Viszeralmedizin 2015;31:47-52.

2 Kromrey M-L, Bülow R, Hübner J, et al: Prospective study on the incidence, prevalence and 5-year pancreatic-related mortality of pancreatic cysts in a population-based study. Gut 2018;67:138-145.

3 Elta GH, Enestvedt BK, Sauer BG, Lennon AM: ACG Clinical Guideline: diagnosis and management of pancreatic cysts. Am J Gastroenterol 2018;113:464-479.

4 Tanaka M, Fernández-Del Castillo C, Kamisawa T, et al: Revisions of international consensus Fukuoka guidelines for the management of IPMN of the pancreas. Pancreatology 2017;17:738-753

5 Jais B, Rebours V, Malleo G, et al: Serous cystic neoplasm of the pancreas: a multinational study of 2622 patients under the auspices of the International Association of Pancreatology and European Pancreatic Club (European Study Group on Cystic Tumors of the Pancreas). Gut 2016;65:305-312.

6 Jones MJ, Buchanan AS, Neal CP, Dennison AR, Metcalfe MS, Garcea G: Imaging of indeterminate pancreatic cystic lesions: a systematic review. Pancreatology 2013;13:436-442.
Tirkes T, Aisen AM, Cramer HM, Zyromski NJ, Sandrasegaran K, Akisik F: Cystic neoplasms of the pancreas; findings on magnetic resonance imaging with pathological, surgical, and clinical correlation. Abdom Imaging 2014;39:1088-1101.

8 Hwang J, Kim YK, Min JH, Jeong WK, Hong SS, Kim H-J: Comparison between MRI with MR cholangiopancreatography and endoscopic ultrasonography for differentiating malignant from benign mucinous neoplasms of the pancreas. Eur Radiol 2018;28:179-187.

9 Thornton GD, McPhail MJW, Nayagam S, Hewitt MJ, Vlavianos P, Monahan KJ: Endoscopic ultrasound guided fine needle aspiration for the diagnosis of pancreatic cystic neoplasms: a meta-analysis. Pancreatology 2013;13:48-57.

10 Brugge WR, Lewandrowski K, Lee-Lewandrowski E, et al: Diagnosis of pancreatic cystic neoplasms: a report of the cooperative pancreatic cyst study. Gastroenterology 2004;126:1330-1336.

11 Smith LA, McKay CJ: The role of endoscopic ultrasound in the management of intraductal papillary mucinous neoplasms: a systematic update. Minerva Med 2016;107:370-380.
2 Tanaka M, Chari S, Adsay V, et al: International consensus guidelines for management of intraductal papillary mucinous neoplasms and mucinous cystic neoplasms of the pancreas. Pancreatology 2006;6:17-32.

13 Tanaka M, Fernández-del Castillo $\mathrm{C}$, Adsay V, et al: International consensus guidelines 2012 for the management of IPMN and MCN of the pancreas. Pancreatology 2012;12:183-197.

14 Anand N, Sampath K, Wu BU: Cyst features and risk of malignancy in intraductal papillary mucinous neoplasms of the pancreas: a meta-analysis. Clin Gastroenterol Hepatol 2013;11:913-921; quiz e59-60.

15 Scheiman JM, Hwang JH, Moayyedi P: American Gastroenterological Association technical review on the diagnosis and management of asymptomatic neoplastic pancreatic cysts. Gastroenterology 2015;148:824-848.e22.

6 Keane MG, Shamali A, Nilsson LN, et al: Risk of malignancy in resected pancreatic mucinous cystic neoplasms. Br J Surg 2018;105:439-446.

17 Law JK, Ahmed A, Singh VK, et al: A systematic review of solid-pseudopapillary neoplasms: are these rare lesions? Pancreas 2014;43:331-337.

18 Krautz C, Nimptsch U, Weber GF, Mansky T, Grützmann R: Effect of hospital volume on in-hospital morbidity and mortality following pancreatic surgery in Germany. Ann Surg 2018;267:411-417. 